\title{
Output-Capacitorless CMOS LDO Regulator Based on High Slew-Rate Current-Mode Transconductance Amplifier
}

\author{
Alireza Saberkari and Rasoul Fathipour \\ Microelectronics Research Lab. \\ Department of Electrical Engineering, University of Guilan \\ Rasht, Iran \\ a_saberkari@guilan.ac.ir
}

\author{
Herminio Martínez; Alberto Poveda and Eduard Alarcón \\ Department of Electronics Engineering \\ Technical University of Catalunya (UPC). BarcelonaTech \\ Barcelona, Spain \\ \{herminio.martinez; eduard.alarcon\}@upc.edu
}

\begin{abstract}
A low quiescent current output-capacitorless CMOS LDO regulator based on a high slew-rate current-mode transconductance amplifier (CTA) as an error amplifier is presented. Load transient characteristic of the proposed LDO is improved even at low quiescent currents, by using a local common-mode feedback (LCMFB) in the proposed CTA. This provides an increase in the order of transfer characteristic of the circuit, thereby enhancing the slew-rate at the gate of pass transistor. The proposed CTA-based LDO topology has been designed and post-layout simulated in HSPICE, in a $0.18 \mu \mathrm{m}$ CMOS process to supply a load current between 0-100 $\mathrm{mA}$. Postlayout simulation results reveal that the proposed LDO is stable without any internal compensation strategy and with on-chip output capacitor or lumped parasitic capacitances at the output node between 10-100 $p F$.
\end{abstract}

\section{INTRODUCTION}

Low-dropout regulators (LDO) are widely used either alone in many integrated power management circuits or as post regulators of switching converters, as they can provide wideband and reliable regulated low noise supply voltage for noise-sensitive analog and RF loads [1]. The typical structure of an LDO consists of an error amplifier, a pass transistor controlled by the error amplifier, a feedback network, and an output capacitor. Most of conventional LDOs use a large offchip capacitor for stability requirements [1, 2]. However, large capacitors can not be implemented as on-chip capacitors. Therefore, output-capacitorless LDOs are needed for SoC applications. However, doing so will cause that the distance between poles at the output node of the LDO and the gate of the pass transistor decreases and, thus, the stability of the circuit worsens, significantly. Additionally, it will increase the output voltage deviations, $\Delta V_{\text {out }}$, when a sudden change occurs in the load current. Therefore, having a stable output-capacitorless LDO with small $\Delta V_{\text {out }}$ and proper settling time $\left(T_{\text {settle }}\right)$ is a design target.

Some papers in connection with output capacitorless LDOs have been reported in recent years [3-7]. The reported LDO in [3] has an ultra fast transient response characteristic with an on-chip output capacitor of $0.6 n F$, which occupies a large silicon area. Moreover, it consumes significant high quiescent current of $6 \mathrm{~mA}$ and, thus, it is not appropriate for low power design. In [4], a slew-rate enhancement stage is used between the output of LDO and the gate of pass transistor which increases the slew-rate at the gate node of the pass transistor and provides proper transient response. Similarly, in [5], a capacitor multiplier stage is used between these two nodes in order to provide enough distance between the dominant and non-dominant poles and also minimize the effect of feedback loop-bandwidth limitation, improving the dynamic performance of the LDO. However, these solutions add more power consumption. LDOs based on the flipped voltage follower (FVF) were introduced in $[6,7]$ which suffer from weak load and line regulation and the need to have minimum load current for stability.

The transient response of an LDO is determined by both the loop gain bandwidth and the slew-rate at the gate of pass transistor $\left(S R_{G}\right)$ [2]. Increasing the bandwidth makes the compensation process more difficult and, therefore, the bandwidth of LDOs is generally low. Additionally, if $S R_{G}$ is much slower than the gain-bandwidth product, transient voltage spikes appear at the output voltage node during fast load transient [1]. Thus, $S R_{G}$ should be increased in order to have a proper transient response. Using a high slew-rate current-mode error amplifier instead of a voltage-mode one, which suffers from limited slew-rate, can improve the transient response while also decreasing power consumption. In [8], a class- $A B$ push-pull amplifier was used as error amplifier to source and sink more current for charging and discharging the gate capacitor $\left(C_{g}\right)$ of the pass transistor during the transient event. However, in low power mode, this amplifier will be capable to generate a considerable current into the output load only if a relatively large input voltage is applied to it. This fact causes high output voltage deviations in the LDO transient response. Additionally, the LDO output current range is between $50 \mu \mathrm{A}$ to $50 \mathrm{~mA}$ and the stability of the regulator goes down significantly for output current less than $50 \mu A$.

This paper presents an output-capacitorless LDO based on a new class- $A B$ current-mode transconductance amplifier (CTA) with capability of generating a considerable current even if the input voltage is not very large. Using this circuit as an error amplifier in the proposed LDO, it results in a 
proper transient response with small $\Delta V_{\text {out }}$ even for low quiescent currents.

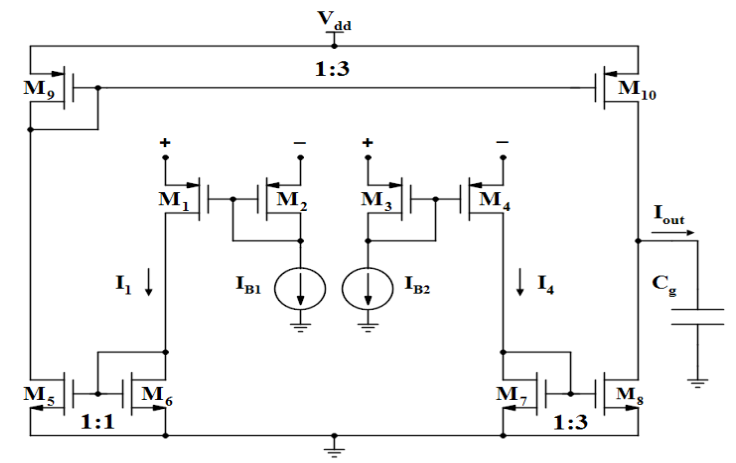

Figure 1. Class-AB transconductance amplifier used in [8]

\section{PROPOSED CTA ERROR AMPLIFIER}

Slew-rate $\left(S R_{G}\right)$ in a class-A OTA is proportional to the maximum bias current that the output capacitor can sink or source. However, increasing the bias current in order to increase $S R_{G}$ will cause more static power loss. Therefore, using a class-AB amplifier instead of class-A can eliminate this limitation. Fig. 1 shows the class- $A B$ transconductance amplifier which was applied in the LDO reported in [8]. Applying the differential voltage $V_{i d}$ to the input nodes, will cause that currents $I_{1}$ and $I_{4}$ always change in different directions. Assuming all transistors operate in the saturation region and neglecting body and channel length modulation effects, the large signal analysis can be derived as:

$$
\begin{gathered}
I_{1}=\frac{\beta_{1}}{2}\left(V_{i d}+V_{S G 2}-\left|V_{t p}\right|\right)^{2}=\frac{\beta_{1}}{2}\left(V_{i d}+\sqrt{\frac{2 I_{B 1}}{\beta_{2}}}\right)^{2} \\
I_{4}=\frac{\beta_{4}}{2}\left(-V_{i d}+V_{S G 3}-\left|V_{t p}\right|\right)^{2}=\frac{\beta_{4}}{2}\left(-V_{i d}+\sqrt{\frac{2 I_{B 2}}{\beta_{3}}}\right)^{2}
\end{gathered}
$$

in which $\beta=\mu_{p} C_{o x}(W / L)$ and $V_{t p}$ is the threshold voltage of input PMOS transistors. According to the aspect ratios of current mirrors and neglecting channel length modulation effect, the output current is $I_{\text {out }}=3 I_{1}-3 I_{4}$. Thus, the transfer characteristic (i.e., $I_{\text {out }}$ versus $V_{i d}$ ) is a second order function of $V_{i d}$. If one can increase the order of the transfer characteristic without decreasing the speed of signal path, this will enhance the slew-rate for the same $V_{i d}$. Using local common-mode feedback (LCMFB) technique can be a suitable approach for this purpose [9, 10]. Fig. 2 shows the proposed current-mode transconductance amplifier (CTA) in which LCMFB technique is used to enhance the slew-rate of the amplifier. When no differential voltage is applied to the input ports, currents $I_{1}$ and $I_{4}$ are equal to $I_{B 1}=I_{B 2}=I_{B}$. In this situation no current flows through $R_{1}$ and $R_{2}$. After applying a differential voltage of $V_{i d}$ to the input ports, a differential current $I_{d}=I_{1}-I_{4}$ is created and the currents through resistors will be $I_{R}=\left(I_{1}-I_{4}\right) / 2$. The drain current of transistors $M_{6}$ and $M_{7}$ will be a common-mode current $I_{c m}=\left(I_{1}+I_{4}\right) / 2$. Therefore, the gate and drain voltages of $M_{6}$ and $M_{7}$ will change as follows:

$$
V_{d 6}=V_{g 6,7}+R_{1} I_{d} / 2 \quad V_{d 7}=V_{g 6,7}-R_{2} I_{d} / 2 \quad V_{g 6,7}=V_{t n}+\sqrt{\frac{2 I_{c m}}{\beta_{6,7}}}
$$

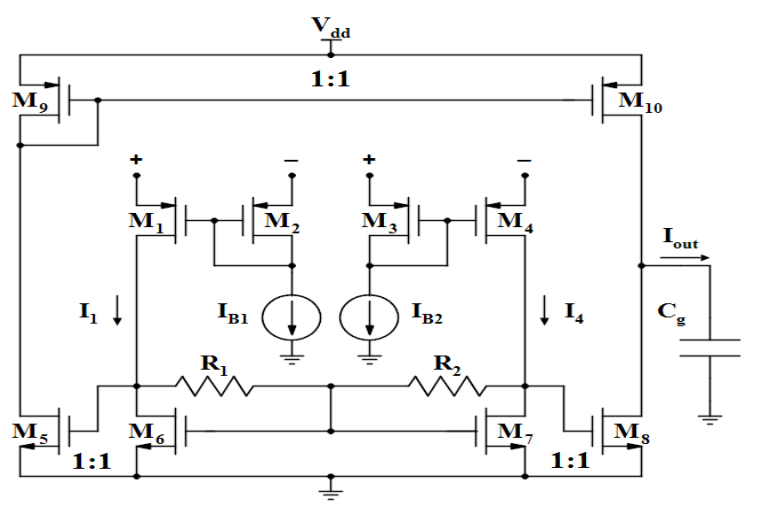

Figure 2. The proposed CTA.

The resistors $R_{1}$ and $R_{2}$ act as a common-mode feedback circuit [11] and control the common-mode voltage level at the drain of $M_{6}$ and $M_{7}$ without altering the differential mode behavior of the circuit. According to (2), the currents of transistors $M_{5}$ and $M_{8}$ are, respectively:

$$
\begin{gathered}
I_{5}=\frac{\beta_{5}}{2}\left(V_{g 6,7}+\frac{R_{1} I_{d}}{2}-V_{t n}\right)^{2}=\frac{\beta_{5}}{2}\left(\sqrt{\frac{2 I_{c m}}{\beta_{6,7}}}+\frac{R_{1} I_{d}}{2}\right)^{2} \\
I_{8}=\frac{\beta_{8}}{2}\left(V_{g 6,7}-\frac{R_{2} I_{d}}{2}-V_{t n}\right)^{2}=\frac{\beta_{8}}{2}\left(\sqrt{\frac{2 I_{c m}}{\beta_{6,7}}}-\frac{R_{2} I_{d}}{2}\right)^{2}
\end{gathered}
$$

Assuming $M_{9}$ and $M_{10}$ are similar, the output current is $I_{\text {out }}=I_{5}-I_{8}$. For $V_{i d}>0$, the output current is $I_{\text {out }} \approx I_{5}$, and, similarly, for $V_{i d}<0, I_{\text {out }} \approx-I_{8}$. Therefore, when $V_{\text {id }} \neq 0$, the output current is equal to:

$$
I_{\text {out }}=I_{5}-I_{8} \approx \pm \frac{\beta_{5,8}}{2}\left(\sqrt{\frac{2 I_{c m}}{\beta_{6,7}}}+\frac{R_{1,2}\left|I_{d}\right|}{2}\right)^{2}
$$

Considering the second order relationship between $\mathrm{I}_{\mathrm{d}}$ and $V_{i d}$ according to (1), the transfer characteristic of the proposed amplifier $\left(I_{\text {out }}\right.$ versus $\left.V_{i d}\right)$ is a $4^{\text {th }}$ order function of $V_{i d}$. As a consequence, the circuit can enhance $S R_{G}$ by generating a considerable current into the output.

\section{PROPOSED LDO}

The transistor-level schematic of the proposed LDO is shown in Fig. 3. It consists of the proposed CTA as the error amplifier, the pass transistor $M_{p}$, the on-chip output capacitor, and the reference buffer. The aspect ratio of the pass transistor has been set to $W / L=6000 \mu \mathrm{m} / 0.18 \mu \mathrm{m}$ to provide a $100-m A$ load current at $200 \mathrm{mV}$ dropout voltage. The LDO is configured in a unity feedback structure to have the output voltage equal to the reference one. When an error between these voltages is sensed by the error amplifier, the CTA generates a suitable current to source or sink the gate capacitance of $M_{p}$. For example, in case that the load current increases, the output voltage will drop. Thus, the drain current of $M_{4}$ increases and that of $M_{1}$ decreases. It will cause the increase of the drain terminal voltage of $M_{7}$ and the decrease of the drain voltage of $M_{6}$. Therefore, the drain current of $M_{8}$ increases and those of $M_{5}, M_{9}$, and $M_{10}$ decrease and, hence, the gate capacitance of $M_{p}$ is discharged so that more drain current is sourced to the load, increasing the output voltage. An analogous dual mechanism occurs when the load current decreases. 


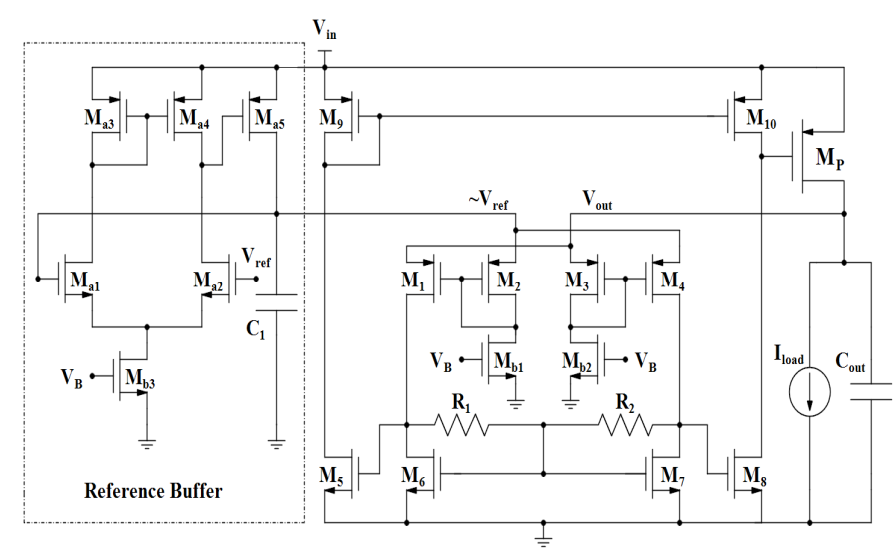

Figure 3. Schematic of the proposed LDO

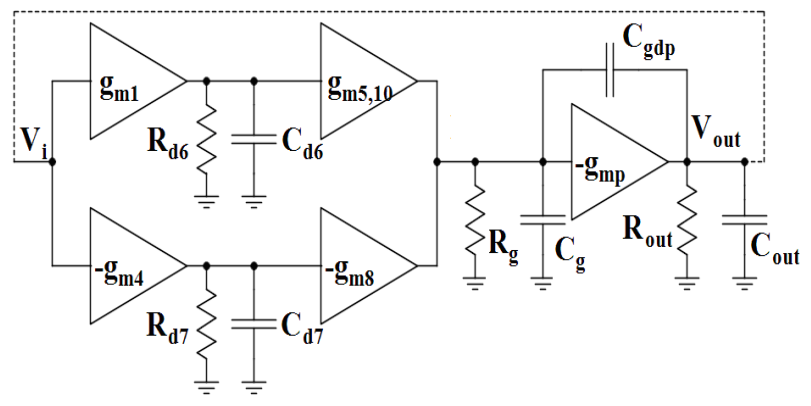

Figure 4. Small signal model of the proposed LDO

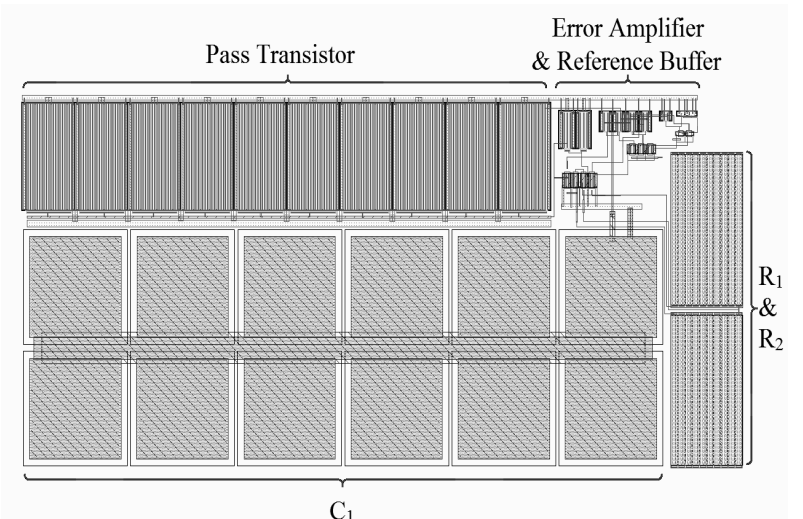

Figure 5. Layout of the proposed LDO

The small signal model of proposed LDO is shown in Fig. 4 , in which $g_{m i}, R_{d i}$, and $C_{d i}$ are the transconductance, output resistance, and capacitance at the drain terminal of transistor $M_{i}$, respectively. Also $R_{g}-C_{g}$ and $R_{\text {out }}-C_{\text {out }}$ are the equivalent resistance and capacitance at the gate and drain terminals of $M_{p}$. Carrying out small signal analysis on the circuit, the DC gain, and pole-zero positions are as bellow:

$$
\begin{gathered}
A_{0} \approx 2 g_{m 1} R_{d 6} g_{m 8} R_{g} g_{m p} R_{\text {out }} \\
p_{1}=\frac{-1}{R_{g} C_{g}} \quad p_{2}=\frac{-1}{R_{\text {out }} C_{\text {out }}} \quad p_{3}=\frac{-1}{R_{d 6} C_{d 6}} \quad z_{1}=\frac{g_{m p}}{C_{\text {gdp }}} \\
\text { Considering } \quad R_{d 6}=R_{d 7} \approx R_{1,2}, \quad R_{g}=r_{\text {os }} \| r_{\text {o10 }},
\end{gathered}
$$

$R_{\text {out }} \approx 1 / g_{m 1} \| r_{o p}$, and assuming $C_{g}, C_{\text {out }}>>C_{d 6}, C_{g d p}$, the pole $P_{3}$ and the zero $Z_{1}$ will be located at frequencies higher than

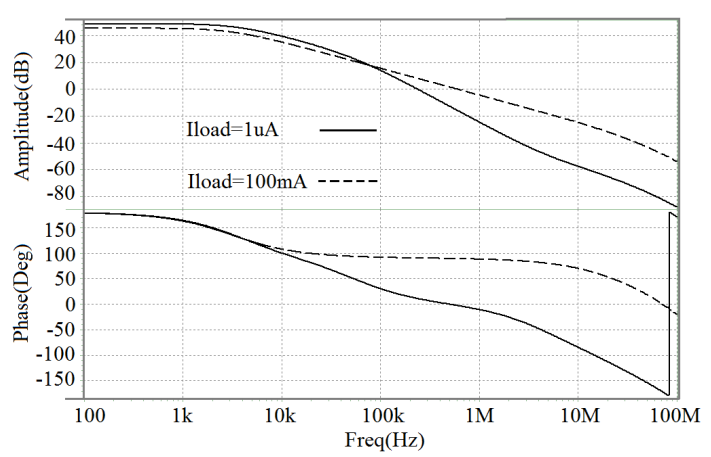

Figure 6. Open loop AC response of the proposed LDO

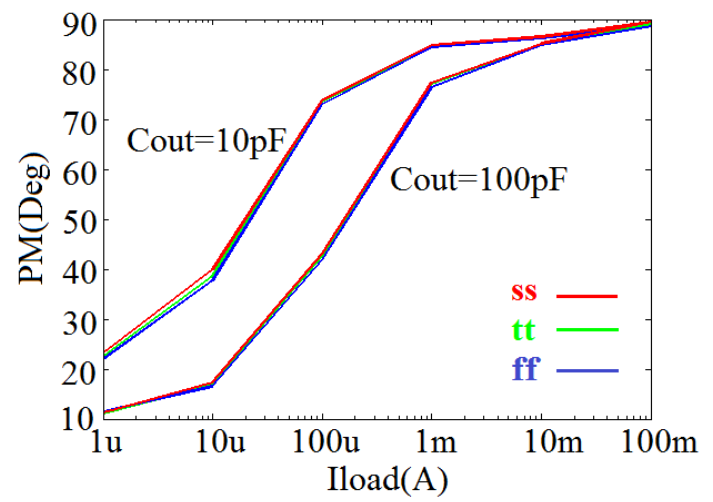

Figure 7. Phase margin of the proposed LDO for different load currents, output capacitors, and process variation

two other poles and the unity gain frequency $\left(\omega_{T}\right)$. Therefore, it is possible to model the loop gain as a transfer function with two poles at frequencies below $\omega_{T}$. With an increase in the load current or for a small output capacitor, the distance between the poles becomes wider and, thus, the phase margin will be enhanced. Moreover, if $R_{1}$ and $R_{2}$ are voltagecontrolled resistors (transistors biased in the triode region), the phase margin can be adjusted by changing the resistors for a certain lumped capacitance at the output.

\section{CIRCUIT CHARACTERIZATION}

The proposed LDO topology has been designed and laid out, as shown in Fig. 5, to source the nominal load current between $0-100 \mathrm{~mA}$ and the obtained performance metrics correspond to HSPICE post simulations in a $0.18 \mu \mathrm{m}$ CMOS process. In order to test the LDO for different values of output capacitor, $C_{\text {out }}$ is not included in the layout and the core area of the chip without the output capacitor is $110 \times 230$ $\mu \mathrm{m}^{2}$. The dropout voltage of the LDO is set to $200 \mathrm{mV}$ for 1.2-2 $V$ input voltage $V_{\text {in }}$. The total quiescent current of the LDO, including the current consumed by the reference buffer circuit, is only $3.7 \mu \mathrm{A}$. Fig. 6 shows the simulated open loop AC response of the proposed LDO for light and heavy load currents with $C_{\text {out }}=100 \mathrm{pF}$. For an accurate $\mathrm{AC}$ response analysis, the values of phase margin for different load currents and output capacitors by considering the effect of process variation are shown in Fig. 7. As it can be seen, even under the worst condition (large output capacitor and light load current) the proposed LDO is stable and has still an adequate phase margin and has a less sensitivity to the process variation. 


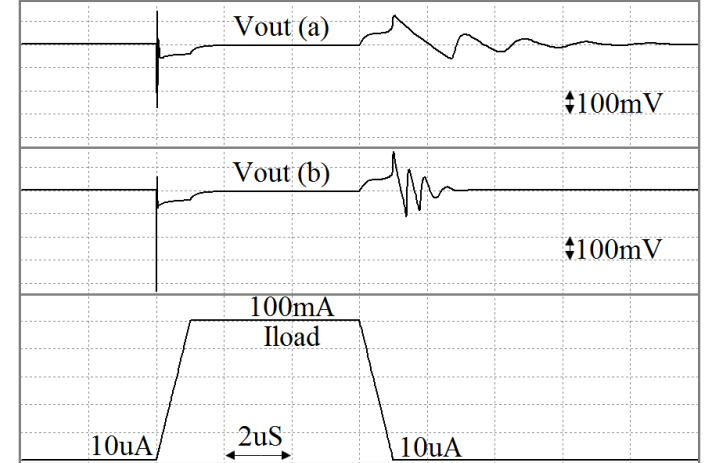

Figure 8. Load transient of the $\operatorname{LDO}$ (a) $C_{\text {out }}=100 \mathrm{pF}$, (b) $C_{\text {out }}=10 \mathrm{pF}$

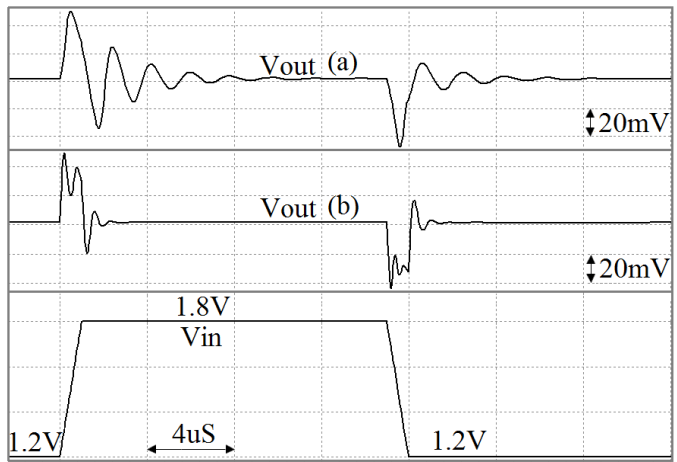

Figure 9. Line transient of the $\mathrm{LDO}$ (a) $C_{\text {out }}=100 \mathrm{pF}$, (b) $C_{\text {out }}=10 \mathrm{pF}$

Fig. 8 shows the load transient response of the LDO for two output capacitors when the load current is changed between light to heavy values with rise and fall times of $1 \mu \mathrm{s}$. As it is obvious, the proposed LDO is stable even for the worst condition and its $T_{\text {settle }}$ and $\Delta V_{\text {out }}$ are $6 \mu \mathrm{s}$ and $277 \mathrm{mV}$, respectively. Line transient response of the LDO is shown in Fig. 9, in which the line voltage is changed between $1.2 \mathrm{~V}$ and $1.8 \mathrm{~V}$ with rise and fall times of $1 \mu \mathrm{s}$. It indicates that, for all cases, the LDO has a $T_{\text {settle }}$ and $\Delta V_{\text {out }}$ less than $9 \mu s$ and 47 $m V$, respectively.

Table I provides a performance comparison between the proposed LDO and recent works. In order to have a fair comparison, the other LDOs are simulated in HSPICE with the parameters mentioned in the papers. The figure of merit $\left(F O M=\Delta V_{\text {out }} C_{\text {out }} I_{Q} / I_{\text {out, } \max }^{2}\right)$ used in [3] is adopted here to compare the transient response of different LDOs. A lower FOM implies better transient response achieved by the LDO. Notice that using a high slew-rate CTA with very low quiescent current in the proposed LDO helps to achieve lower FOM in comparison to the other reported alternatives.

\section{CONCLUSION}

An output-capacitorless CMOS LDO regulator based on a high slew-rate CTA is presented. The load transient characteristic of the LDO is improved by using a LCMFB technique in the proposed CTA which results in an increase of its nonlinear transfer characteristic order. Post-layout simulation results in a $0.18 \mu \mathrm{m}$ CMOS process show that the proposed LDO is stable for a wide load current range between 0-100 $m A$ without any internal compensation capacitor and with output capacitor in the range of 10-100 $p F$ while its total quiescent current is only $3.7 \mu \mathrm{A}$.
TABLE I. PERFORMANCE COMPARISON

\begin{tabular}{|c|c|c|c|c|c|}
\hline Parameter & {$[4]$} & {$[5]$} & {$[6]$} & {$[8]$} & This Work \\
\hline Tech $[\mu \mathrm{m}]$ & 0.35 & 0.35 & 0.35 & 0.18 & $\mathbf{0 . 1 8}$ \\
\hline$V_{I N}[\mathrm{~V}]$ & 1.8 & 3 & 1.2 & 1.2 & $\mathbf{1 . 2}$ \\
\hline$V_{\text {OUT }}[\mathrm{V}]$ & 1.6 & 2.8 & 1 & 1 & $\mathbf{1}$ \\
\hline$I_{\text {OUT }}[\mathrm{mA}]$ & 100 & 50 & 50 & 50 & $\mathbf{1 0 0}$ \\
\hline$I_{Q}[\mu \mathrm{A}]$ & 20 & 66 & 95 & 1.2 & $\mathbf{3 . 7}$ \\
\hline$C_{\text {OUT }}[\mathrm{pF}]$ & 100 & 100 & 20 & 100 & $\mathbf{1 0 0}$ \\
\hline$T_{\text {settle }}[\mu s]$ & $\approx 8$ & $\approx 4$ & $\approx 1.4$ & $\approx 4.4$ & $\approx \mathbf{6}$ \\
\hline$\Delta V_{\text {OUT }}[\mathrm{mV}]$ & 100 & 110 & 200 & 490 & $\mathbf{2 7 7}$ \\
\hline CE $(\%)$ & 99.98 & 99.86 & 99.81 & 99.99 & $\mathbf{9 9 . 9 9}$ \\
\hline FOM $[f s]$ & 20 & 290 & 152 & 23.5 & $\mathbf{1 0 . 2}$ \\
\hline
\end{tabular}

ACKNOWLEDGMENTS

This work has been partially supported by Micro-ISTI, and the Spanish Ministry of Science and Innovation thanks to projects TEC2010-15765, and RUE CSD2009-00046 (Consolider-Ingenio 2010).

\section{REFERENCES}

[1] A. Saberkari, E. Alarcon, and Sh. B. Shokouhi, "Fast Transient Current-Steering CMOS LDO Regulator Based on Current Feedback Amplifier," Integration, the VLSI Journal (2012), DOI: 10.1016/j.vlsi.2012.02.001.

[2] K. N. Leung and Y. S. Ng, "A CMOS Low-Dropout Regulator with a Momentarily Current-Boosting Voltage Buffer," IEEE Trans. Circuits Syst. I, vol. 57, no. 9, pp. 2312-2319, Sept. 2010.

[3] P. Hazucha, T. Karnik, B. Bloechel, C. Parsons, D. Finan, and S Borkar, "Area-Efficient Linear Regulator with Ultra-Fast Load Regulation," IEEE J. Solid-State Circuits, vol. 40, no. 4, pp. 933-940, Apr. 2005

[4] E. N. Y. Ho and P. K. T. Mok, "A Capacitor-Less CMOS Active Feedback Low-Dropout Regulator with Slew-Rate Enhancement for Portable On-Chip Applications," IEEE Trans. Circuits Syst. II, vol. 52, no. 2, pp. 80-84, Feb. 2010.

[5] R. J. Milliken, J. Silva-Martinez, and E. Sanchez-Sinencio, "Full OnChip CMOS Low-Dropout Voltage Regulator," IEEE Trans. Circuits Syst. I, vol. 54, no. 9, pp. 1879-1890, Sept. 2007.

[6] T. Y. Man, K. L. Leung, C. Y. Leung, P. K. T. Mok, and M. Chan, "Development of Single-Transistor-Control LDO Based on Flipped Voltage Follower for SoC," IEEE Trans. Circuits Syst. I, vol. 55, no. 5, pp. 1392-1401, Jun. 2008.

[7] J. Guo and K. N. Leung, "A 6- $\mu \mathrm{W}$ Chip-Area-Efficient OutputCapacitorless LDO in 90-nm CMOS Technology," IEEE J. Solid-State Circuits, vol. 45, no. 9, pp. 1896-1905, Sept. 2010.

[8] T. Y. Man, P. K. T. Mok, and M. Chan, "A High Slew-Rate Push-Pull Output Amplifier for Low-Quiescent Current Low-Dropout Regulators With Transient-Response Improvement," IEEE Trans. Circuits Syst. II, vol. 54, no. 9, pp. 755-759, Sept. 2007.

[9] A. J. Lopez-Martin, S. Baswa, J. Ramirez-Angulo, and R. G. Carvajal "Low-Voltage Super Class-AB CMOS OTA Cell with Very High Slew- Rate and Power Efficiency," IEEE J. Solid-State Circuits, vol. 40, no. 5, pp. 1068-1077, May 2005.

[10] J. Ramirez-Angulo and M. Holmes, "Simple Technique Using Local CMFB to Enhance Slew-Rate and Bandwidth of One-Stage CMOS OpAmps," Electron. Lett., vol. 38, no. 23, pp. 1409-1411, Nov. 2002.

[11] A. Saberkari, R. Fathipour, and H. Saberkari, "Design and Analysis of a Continuous-Time Common-Mode Feedback Circuit Based on Differential-Difference Amplifier," Research J. Applied Sciences Engineering Technology, vol. 4, no. 5, pp. 452-457, Mar. 2012. 